\title{
Use of tracheal palpation to assure correct placement of an endotracheal tube: Letter One
}

\author{
Devanand Mangar, MD · Collin Sprenker, BS • \\ Rachel Karlnoski, PhD $\cdot$ Enrico Camporesi, MD
}

Received: 30 December 2013/Accepted: 13 March 2014/Published online: 25 April 2014

(c) Canadian Anesthesiologists' Society 2014

\section{To the Editor,}

We read with interest the recent paper by McKay et al. regarding the assessment of endotracheal tube (ETT) depth by tracheal palpation (TP). ${ }^{1}$ The authors reported that the usage of TP resulted in more correct ETT placements $(77 \%$ vs $57 \% ; P=0.037)$ when compared with the practice of using a fixed distance at the teeth $(21 \mathrm{~cm}$ for women, $23 \mathrm{~cm}$ for men). The authors defined correct placement as an ETT tip positioned $>2.5 \mathrm{~cm}$ from the carina and $>3.5 \mathrm{~cm}$ below the vocal cords. We commend the authors for their important work but caution the clinical implications of the reported results.

Our primary concern with the study design is the comparison of the TP method with the 21/23 method for correct ETT placement. Many clinicians consider the 21/23 method as an inefficient method that does not account for anatomical neck variations amongst patients. Several studies have now shown that this method results in the incorrect placement of ETTs and should not be considered the gold standard for locating an ideal tracheal depth. ${ }^{2-5}$ Although not routinely performed, in our view, the gold standard for locating the correct ETT insertion depth for endobronchial intubation is via flexible bronchoscopy. The authors did utilize flexible bronchoscopy to confirm endobronchial depth (completed on $92 \%$ of patients) and found $71(77 \%)$ ETTs in the correct position when utilizing

D. Mangar, MD - C. Sprenker, BS · R. Karlnoski, PhD .

E. Camporesi, MD

Florida Gulf to Bay Anesthesiology Associates, LLC, Tampa, FL, USA

R. Karlnoski, PhD - E. Camporesi, MD ( $₫)$

Department of Surgery, University of South Florida, Tampa,

FL, USA

e-mail: ecampore@health.usf.edu the TP method. It would be interesting for the authors to report whether a 33\% ETT malposition rate is a clinically insignificant failure rate.

At our institution, we practice routine confirmation of ETT depth via a flexible bronchoscope in all patients predetermined by the anesthesiologist to undergo a difficult tracheal intubation (body mass index $[\mathrm{BMI}] \geq 30$, Mallampati score $\geq 3$ ). Indeed, the authors attest that confidently feeling (and thus identifying) the ETT was in only $65 \%$ of patients with the palpation method. We assume patients with large necks and an increased BMI were excluded from this exploratory study. Further research to confirm the efficacy of the TP method would require a randomized clinical trial where potential patients with a large BMI and neck size could be enrolled.

Conflicts of interest None declared.

\section{References}

1. McKay WP, Klonarakis J, Pelivanov V, O'Brien JM, Plewes $C$. Tracheal palpation to assess endotracheal tube depth: an exploratory study. Can J Anesth 2014; 61: 229-34.

2. Ong KC, A'Court GD, Eng P, Ong YY. Ideal endotracheal tube placement by referencing measurements on the tube. Ann Acad Med Singapore 1996; 25: 550-2.

3. Hwang JY, Rhee KY, Kim JH, Park YS, Han SH. Methods of endotracheal tube placement in patients undergoing pelviscopic surgery. Anaesth Intensive Care 2007; 35: 953-6.

4. Evron $S$, Weisenberg $M$, Harow E, et al. Proper insertion depth of endotracheal tubes in adults by topographic landmarks measurements. J Clin Anesth 2007; 19: 15-9.

5. Mangar D, Sprenker CJ, Karlnoski RA, et al. Migration of polyurethane high-volume low-pressure cuffed endotracheal tubes after neck flexion and extension. J Anesth 2013; 27: 633-4. 